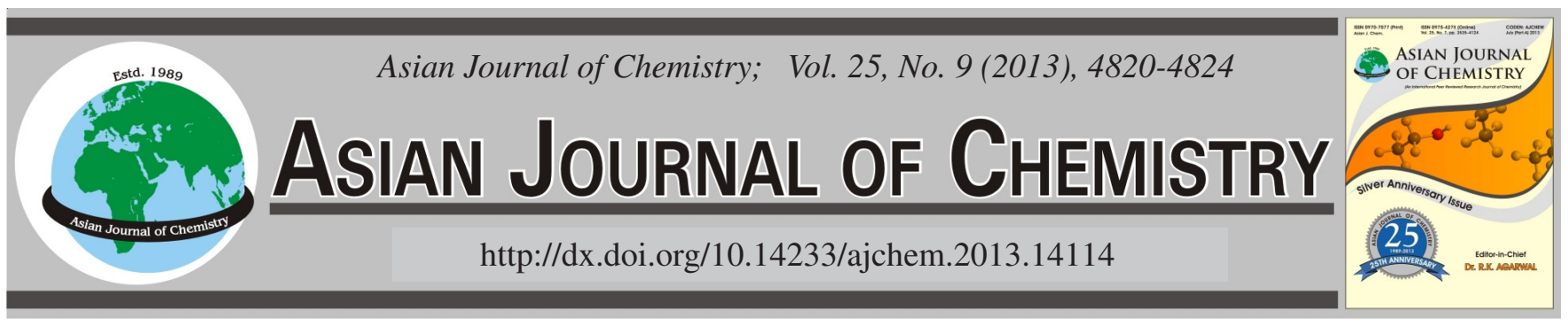

\title{
Determination of Dapiprazole Hydrochloride in the Presence of Process and Degradation Impurities by a Validated Stability Indicating RP-HPLC Method
}

\author{
K. Jaya Prasanthi and B. Syama Sundar ${ }^{*}$
}

Department of Chemistry, Acharya Nagarjuna University, Nagarjuna Nagar, Guntur-522 510, India

*Corresponding author: E-mail: profbsyamsundar@yahoo.co.in

Dapiprazole hydrochloride is an $\alpha$-adrenergic blocking agent. Chemically, it is 5,6,7,8-tetrahydro-3-[2-(4- $o$-tolyl-1-piperazinyl)ethyl]-striazolo[4,3-a]pyridine hydrochloride, which is widely used in ophthalmic practice. A stability-indicating RP-HPLC method has been developed and validated which can separate and accurately quantitate dapiprazole related compounds. The method was successfully validated in accordance to ICH guidelines acceptance criteria for system suitability, specificity, linearity, range, precision, accuracy, limits of detection and quantification for the impurities and robustness, following the ICH guidelines. Therefore, the proposed method was suitable for the simultaneous determination of dapiprazole and its process related and degreadation impurities. Finally, the applicability of the method was evaluated in commercial dosage form analysis as well as in stability studies.

Key Words: Dapiprazole, Degradants, Validation, Stability indicating HPLC method.

\section{INTRODUCTION}

Dapiprazole hydrochloride is an $\alpha$-adrenergic blocking agent ${ }^{1,2}$. Chemically, it is 5,6,7,8-tetrahydro-3-[2-(4-o-tolyl1-piperazinyl)ethyl]-s-triazolo[4,3-a] pyridine hydrochloride ${ }^{3}$ (empirical formula $\mathrm{C}_{19} \mathrm{H}_{27} \mathrm{~N}_{5} \cdot \mathrm{HCl}$, m.w. 361.93). It is a sterile, white, lyophilized powder soluble in water. Dapiprazole hydrochloride ophthalmic solution is indicated in the treatment of iatrogenically induced mydriasis produced by adrenergic (phenylephrine) or parasympatholytic (tropicamide) agents ${ }^{4}$. The aim of this paper was to develop validate a simple and reliable HPLC-UV method for the simultaneous determination of dapiprazole and its process related impurity-A. Besides the simple route of synthesis for dapiprazole, we have observed a potential impurity (Imp-A) in our synthetic process and some unknown impurities in forced degradation. Hence, a stabilityindicating HPLC method for determination of dapiprazole in presence of process and degradation impurities was developed and validated as per International Conference on Harmonization (ICH) guidelines. This manuscript describes the development and validation, in accordance with ICH guidelines, of a rapid, economical, precise and accurate stability-indicating isocratic reversed-phase HPLC method for analysis of dapiprazole in the presence of its process related impurity. This paper also deals with the forced degradation of dapiprazole under the stress conditions acidic and basic hydrolysis, oxidation, heat and light and validation of the method for accurate quantification of dapiprazole in the drug substance. To the best of our knowledge, no analytical methods are currently available to separate and quantitate the known process related compounds of dapiprazole API. Furthermore, there is no stability-indicating HPLC method reported in the literature that can adequately separate and accurately quantitate dapiprazole and degradation compounds. Chemical structures of dapiprazole and it process related impurity was shown below. (Fig. 1(a)) and the synthetic route, in which the process related impurity has been formed, is shown in Fig. 1(b).

\section{EXPERIMENTAL}

Waters Alliance 2695 separation module (Waters Corporation, Milford, USA) equipped with $2489 \mathrm{UV} /$ visible detector or 2998 PDA detector (for specificity and forced degradation studies) with Empower 2 software was used for the analysis. The chromatographic and the integrated data were recorded using HP-Vectra (Hewlett Packard, Waldbronn, Germany) computer system. Chromatographic separation of dapiprazole and its process related impurity was achieved on Inertsil ODS $\mathrm{C}_{18}-3 \mathrm{~V}(250 \mathrm{~mm} \times 4.6 \mathrm{~mm}, 5 \mu$ particle size $)$. The $\mathrm{pH}$ measurements were carried out with Elico, model LI 120, pH meter equipped with a combined glass-calomel electrode calibrated using standard buffer solutions of $\mathrm{pH}$ 4.0, 7.0 and 9.2.

All the reagents were of analytical-reagent grade unless stated otherwise. Glass-distilled and deionized water (Nanopure, Barnstead, USA), HPLC-grade acetonitrile, potassium dihydrogen 
orthophosphate, sodium hydroxide, hydrochloric acid, hydrogen peroxide and orthophosphoric acid (SD Fine Chem, Mumbai, India) were used. Active pharmaceutical ingredient of dapiprazole (reference standard) and its process related substances viz. Impurity, 1-(o-tolyl)-piperizine were a kind of gift from Hetero Drugs Pvt. Ltd., Hyderabad.

Chromatographic conditions: Separation was achieved on a Inertsil-ODS- $\mathrm{C}_{18}$-3VS column $(250 \mathrm{~mm} \times 4.0 \mathrm{~mm}, 5 \mu \mathrm{m})$ using an isocratic mode by the mobile phase consists of a mixture of buffer and acetonitrile in the ratio of $85: 15 \mathrm{v} / \mathrm{v}$ was at pH-3.2 adjusted with $10 \%$ orthophosphoric acid in water. The column was then re-equilibrated for $10 \mathrm{~min}$ with mobile phase. The flow rate of the mobile phase was $1 \mathrm{~mL} / \mathrm{min}$ and the total elution time, including the column re-equilibration, was $c a .0 .5 \mathrm{~h}$. The UV detection wavelength was carried at $205 \mathrm{~nm}$. The injection volume was $20 \mu \mathrm{L}$ and experiments were conducted at ambient temperature.

Preparation of system suitability solution (standard solution): Standards of dapiprazole and its process related impurity (20 mg each) were accurately weighed, transferred into $100 \mathrm{~mL}$ volumetric flasks, dissolved in diluents and made up to the mark with the mobile phase to get 200 ppm each of dapiprazole and its process related impurity solution. Transfer the $0.5 \mathrm{~mL}$ of the above stock solution and was adequately diluted up to $100 \mathrm{~mL}$ in volumetric flask with mobile phase to get the concentration of $3 \mu \mathrm{g} / \mathrm{mL}(0.15 \%$ level $)$.

Preparation of sample solutions: Samples of bulk drugs of dapiprazole $(3 \mathrm{mg} / \mathrm{mL}$ ) were prepared as by dissolving $75 \mathrm{mg}$ of dapiprazole in $25 \mathrm{~mL}$ volumetric flask containing diluents solution.
Forced degradation solutions: Weigh accurately $25 \mathrm{mg}$ of dapiprazole sample and transfer into a $50 \mathrm{~mL}$ volumetric flask and make up the volume up to $50 \mathrm{~mL}$ with mobile phase to get the concentration of $500 \mu \mathrm{g} / \mathrm{mL}$ of dapiprazole standard solution.

Method validation: Validation of the developed method for the determination of dapiprazole and its process related mpurity was performed according to the ICH guidelines with standards and API. Thus, system suitability along with method selectivity, specificity, linearity, range, precision, accuracy, limits of detection and quantification for the impurities, short term and long term stability of the analytes in the prepared solutions and robustness were demonstrated.

System suitability: The system suitability was conducted using $1.5 \%(\mathrm{w} / \mathrm{w})$ of the impurity spiked to dapiprazole (3.0 $\mathrm{mg} / \mathrm{mL}$ ) and evaluated by making three replicate injections. The system was suitable for use if the tailing factors for dapiprazole and its impurity were $<1.55$ (observed value is 1.06 ) and the resolution was $>1.90$ (observed value is 7.33).

Specificity: Specificity of a method can be defined as the absence of any interference at retention times of peaks of interest and is normally evaluated by observing the chromatograms of blank samples and samples spiked with the API (active pharmaceutical ingredient) in the presence of all impurities in the mobile phase and the bulk drug. A separate solution of blank, standard and batch samples of dapiprazole were evaluated along with impurity solutions.

Linearity and range: The linearity of dapiprazole and its process related impurity was also studied by preparing standard solutions at seven different levels ranging from 0.75 -
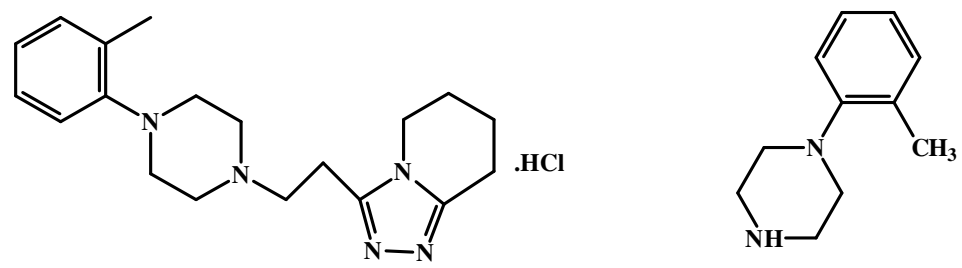

Impurity-A: 1-(o-Tolyl)-piperizine

Fig. 1. (a): Structures of dapiprazole and its impurity

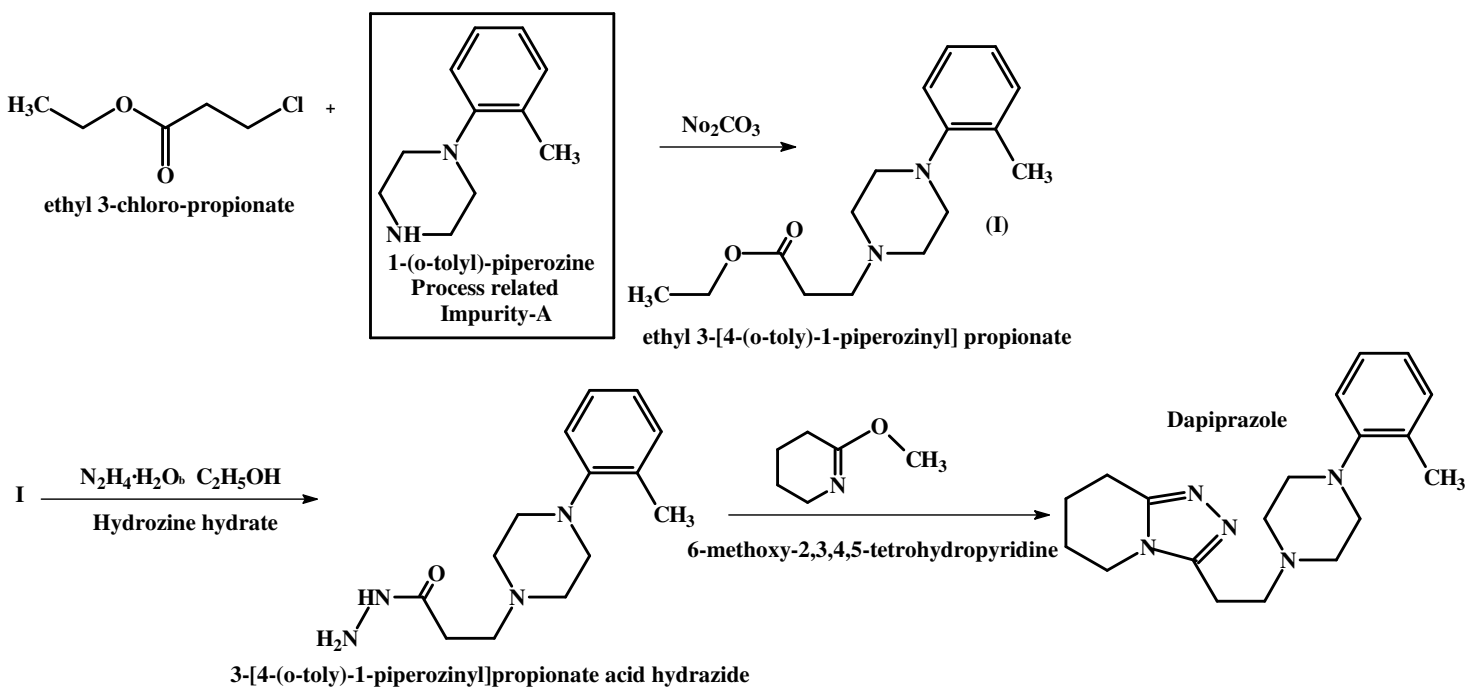

Fig. 1. (b) Synthesis of dapiprazole 
$6 \mu \mathrm{g} / \mathrm{mL}$. The data were subjected to statistical analysis using a linear-regression model; the regression equations and coefficients $\left(r^{2}\right)$ are given in calibration curves of dapiprazole and its process related impurity (o-tolyl piperizine) in Fig. 2.
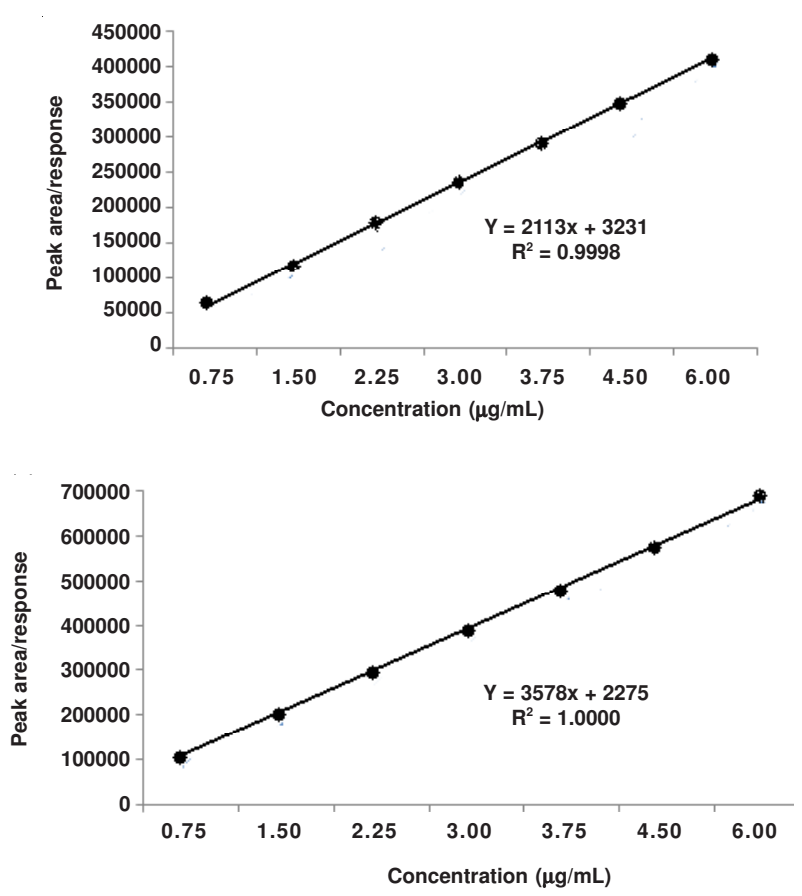

Fig. 2. Calibration graphs of dapiprazole and its impuirity

Precision: Precision as measured by multiple injections of a homogeneous sample (prepared solution) indicates the performance of the HPLC instrument under the chromatographic conditions and day tested. The system precision was conducted using $3.0 \mu \mathrm{g} / \mathrm{mL}$ each of the process related impurity and dapiprazole (i.e., $100 \%$ level) and evaluated by making six replicate injections. The method precision was conducted by using $15 \%$ (w/w) (i.e., $0.45 \mu \mathrm{g} / \mathrm{mL}$ ) of the impurity spiked to dapiprazole (3 mg/mL) (i.e., $100 \%$ level) and evaluated by making six replicate injections.

Accuracy: Accuracy of the method by recoveries for the impurity was determined by analyzing dapiprazole sample solutions spiked with each impurity at five different concentration levels ranging from $25 \%$ (contains $0.75 \mu \mathrm{g} / \mathrm{mL}$ of each of dapiprazole and its process related impurity-A), to $150 \%$ (contains $4.5 \mu \mathrm{g} / \mathrm{mL}$ of each of dapiprazole and its process related impurity-A) in duplicate with respect to specified limit.

LOD and LOQ: The LOD and LOQ were determined for dapiprazole and its process related impurity based on the standard deviation of (SD) of the response and slope (S) of the regression line as per ICH guidelines according to the formulae give below:

$$
\mathrm{LOD}=\frac{3.3 \times \mathrm{SD}}{\mathrm{S}} \quad \mathrm{LOQ}=\frac{10 \times \mathrm{SD}}{\mathrm{S}}
$$

Robustness: The robustness of the method was evaluated through the studies of influence of small and premeditated alteration of analytical parameters. The parameters selected were flow rate $( \pm 10 \%)$, column variation and instrument variation. The above deliberate changes in flow rate are applied at two levels of robustness study that is at upper level and lower level.
Forced degradation studies: Once the chromatographic conditions were selected and the retention of dapiprazole was optimized, preliminary forced degradation studies were conducted using heat, acid and base hydrolysis, light and peroxide. The drug was exposed to extreme conditions to verify its stability under the conditions studied. To demonstrate the degradation of the sample in stress study, sample is prepared and is evaluated at $0 \mathrm{~h}$ and after $48 \mathrm{~h}$.

Acid stress study: Weigh accurately and transfer $c a .300$ mg dapiprazole bulk sample in $20 \mathrm{~mL}$ of $0.1 \mathrm{~N}$ hydrochloric acid. The $0 \mathrm{~h}$-sample solution, has been prepared by taking immediately $5 \mathrm{~mL}$ of the above primary working standard solution and neutralize with $5 \mathrm{~mL}$ of $0.1 \mathrm{~N}$ sodium hydroxide solution and makeup to $25 \mathrm{~mL}$ with diluents in volumetric flask. The acid stress study sample after $48 \mathrm{~h}$ was prepared by taking the $5 \mathrm{~mL}$ of acid hydrolyzed sample solution in $25 \mathrm{~mL}$ volumetric flask and neutralize with $5 \mathrm{~mL}$ of $0.1 \mathrm{~N} \mathrm{NaOH}$ solution and make up to 25 with diluent. The above solutions inject once after system suitability studies.

Base stress studies: Weigh accurately and transfer $c a$. $300 \mathrm{mg}$ dapiprazole bulk sample in $20 \mathrm{~mL}$ of $0.1 \mathrm{~N}$ sodium hydroxide solutions. The zero hour-sample solution, has been prepared by taking immediately $5 \mathrm{~mL}$ of the above primary working standard solution and neutralize with $5 \mathrm{~mL}$ of $0.1 \mathrm{~N}$ hydrochloric acid solution and makeup to $25 \mathrm{~mL}$ with diluents in volumetric flask. The base stress study sample after $48 \mathrm{~h}$ was prepared by taking the $5 \mathrm{~mL}$ of base hydrolyzed sample solution in $25 \mathrm{~mL}$ volumetric flask and neutralize with $5 \mathrm{~mL}$ of $0.1 \mathrm{~N} \mathrm{HCl}$ solution and make up to 25 with diluents. The above solutions were injected into liquid chromatograph, after system suitability studies.

Oxidation stress studies: To demonstrate the degradation of the sample towards oxidation with hydrogen peroxide, the oxidized sample has been prepared at $0 \mathrm{~h}$ and after $48 \mathrm{~h}$. Weigh accurately and transfer $c a .300 \mathrm{mg}$ dapiprazole bulk sample in $20 \mathrm{~mL}$ of $3 \%$ hydrogen peroxide solution. The $0 \mathrm{~h}$ - sample solution, has been prepared by taking immediately $5 \mathrm{~mL}$ of the above primary working standard solution and diluted up to $25 \mathrm{~mL}$ with diluents in volumetric flask. The peroxide stress study sample after $48 \mathrm{~h}$ was prepared by taking the $5 \mathrm{~mL}$ of oxidized sample solution in $25 \mathrm{~mL}$ volumetric flask and dilute up to 25 with diluents. The above solutions inject once after system suitability studies.

Thermal stress studies: To determine stability of dapiprazole towards heat, sample is kept in Petri dish and placed in oven at 40 and $80{ }^{\circ} \mathrm{C}$ up to $48 \mathrm{~h}$. Samples were checked for initial degradation after exposure for $0.5 \mathrm{~h}$ at $40^{\circ} \mathrm{C}$ and at $80{ }^{\circ} \mathrm{C}$. After $48 \mathrm{~h}$, the thermally exposed samples are further used to prepare the testing solution at the strength of $3000 \mathrm{ppm}$ and injected in to the liquid chromatograph.

Photo-degradation studies: To demonstrate the photo degradation of the dapiprazole and effects of UV-irradiation, sample is kept in open Petri dish at Lab light and UV light. Samples were checked for initial degradation after exposure for $0.5 \mathrm{~h}$ at 40 and at $80^{\circ} \mathrm{C}$. After $48 \mathrm{~h}$, the samples are removed from the UV-light cabinet. The light-exposed samples are further used to prepare the testing solution by dilution with mobile phase at the strength of $3000 \mathrm{ppm}$ and injected in to the liquid chromatograph. 


\section{RESULTS AND DISCUSSION}

Optimization of chromatographic conditions: To optimize the chromatographic conditions, different combinations of methanol-water (90:10, 80:20, 70:30, 60:40, 50:50, 40:60, 30:70), acetonitrile-water (90:10, 80:20, 70:30, 60:40, 50:50, 40:60, 30:70) and acetonitrile-potassium dihydrogen phosphate buffer $(90: 10,80: 20,70: 30,60: 40,50: 50,40: 60,30: 70)$ were tested. Acetonitrile with phosphate buffer $(\mathrm{pH} 3.5)$ was preferred because it resulted in a greater response to dapiprazole and also to its related impurity after several preliminary investigatory runs compared with the other mobile phases. The composition, $\mathrm{pH}$ and flow rate of the mobile phase were changed to optimize the separation conditions. Increasing the organic modifier content resulted in a decrease in the retention time of the drug. The effect of the flow rate was studied in the range $0.8-1.2 \mathrm{~mL} / \mathrm{min}$. Higher acetonitrile content and flow rate resulted, as expected, in rapid analysis before dead volume. A high buffer concentration was therefore used at a flow rate of $1 \mathrm{~mL} / \mathrm{min}$, keeping in mind the possibility that potential minor degradation products could appear after stress studies and might co-elute with the drug because of the reduced RT if the flow rate was increased. High flow rates also reduce the life time of both column and pump. Changing the buffer $\mathrm{pH}$ from 3.5-5.5 (by addition of potassium dihydrogen phosphate $(0.01 \mathrm{M})$ or ortho-phosphoric acid) affected the RT and shape of the dapiprazole impurity peak. The effect of $\mathrm{pH}$ on analyte elution was related to the degree of ionization. Reducing the $\mathrm{pH}$ resulted in a shorter $\mathrm{RT}$ of process-related impurity because of ionization of its basic site. A pH of 3.2 was regarded as optimum because at this $\mathrm{pH}$ the analyte peak was sharp and well-defined. The preferred brand of HPLC column should be selected primarily based on the long term stability and lot-to-lot reproducibility. Preliminary development trials have performed with various octadecyl columns $\left(\mathrm{C}_{18}\right.$ columns $)$ of different types and dimensions from different manufacturers were tested for the peak shape and the number of theoretical plates of dapiprazole raw material at $500 \mu \mathrm{g} / \mathrm{mL}$ concentration. Finally by switching to Inertsil ODS- $\mathrm{C}_{18}-3 \mathrm{~V}$ column $(250 \mathrm{~mm}$ $\times 4.6 \mathrm{~mm}, 5 \mu)$ column there was a substantial increase in the theoretical plates ( $c a$. 50000) with a significant improvement in the peak shapes with 1.14 tailing factor. It also produced adequate resolution between dapiprazole and its process related impurity. As a result, it was selected as an optimum one and used throughout this investigation.

Linearity: The calibration curve showed good linearity in the range of $0.75-6.0 \mu \mathrm{g} / \mathrm{mL}$, for dapiprazole and its related substance with correlation coefficient $\mathrm{R}^{2}=$ of 0.9998 and 1.0000, respectively indicating strong correlation (Fig. 2).
Precision: The results of system precision $(\mathrm{RSD} \%=0.40$ for 0.47 for dapiprazole and its process related impurity, respectively), method precision (RSD \% $=1.4$ and 1.0 for dapiprazole and its process related impurity, respectively) are found within the prescribed limit of ICH guidelines (RSD \% $<1 \%$ and $\mathrm{RSD} \%<2 \%$, respectively in case of system precision and method precision.

Accuracy: The accuracy of the method was determined by measurement of recovery. The results of accuracy studies from standard solution and process related impurity were shown in Table-1. The recovery values demonstrated that the method was accurate within the desired range. The results revealed an excellent correlation between the amount added and the amount found.

TABLE-1

ACCURACY STUDIES OF DAPIPRAZOLE IMPURITY-A

Recovery Dapiproazole process related impurity-A

solution at diff. Amount added Amount found Recovery

$\begin{array}{lccc}\text { spiked levels } & (\mathrm{mg} / \mathrm{mL}) & (\mathrm{mg} / \mathrm{mL}) & (\%)\end{array}$

$\begin{array}{llll}25 \% \text { level } & 0.000757 & 0.000775 & 102.37\end{array}$

$\begin{array}{llll}50 \% \text { level } & 0.001515 & 0.001535 & 101.32\end{array}$

$\begin{array}{llll}75 \% \text { level } & 0.002272 & 0.002308 & 101.58\end{array}$

$100 \%$ level $\quad 0.00303 \quad 0.003027 \quad 99.90$

$\begin{array}{llll}125 \% \text { level } & 0.003787 & 0.003805 & 100.47\end{array}$

$\begin{array}{llll}150 \% \text { level } & 0.004545 & 0.004705 & 103.52\end{array}$

Limit of detection and limit of quantification: The detection and quantification limits were evaluated from calibration curves plotted in concentration ranges between 0.75-6.0 $\mu \mathrm{g} / \mathrm{mL}$ of dapiprazole and its process related impurity. The standard drug solutions, for each value of LOD and LOQ concentration were injected 6 times. RSD \% values for the area of replicate injections were calculated. The LOD and LOQ for the dapiprazole process related impurity-A was found to be $1.0 \%(0.03 \mathrm{mcg} /$ $\mathrm{mL})$ and $2.5 \%(0.075 \mathrm{mcg} / \mathrm{mL})$, respectively (Table-2).

Robustness: Robustness study, conducted by deliberate changes in flow rate, different instrument and different batches of column, revealed that there was no significant variation in $\%$ assay, retention time (RT), peak areas and resolution factor (Table-3). The studies indicated no effect on the determination of related substances and selectivity for the test method is sufficiently robust to carry the quantification of impurity in quality assurance of dapiprazole.

Results of forced degradation studies: Subsequently, different forced degradation samples were analyzed. Both the drug and impurity peaks in acid, alkaline, oxidation, thermal and photo-degraded solutions passed the purity test. The chromatogram of $0 \mathrm{~h}$ sample in acid-hydrolysis stress study shows no degradation of dapiprazole indicates stability of the

TABLE-2

LOD AND LOQ DATA FOR IMPURITY-A

\begin{tabular}{|c|c|c|c|c|}
\hline Linearity/range levels (\%) & Impurity-A $(\mu \mathrm{g} / \mathrm{mL})$ & Peak area/response & Impurity-A-Std. deviation & \\
\hline 25 & 0.75 & 84715 & 1330.2 & \\
\hline 50 & 1.5 & 173940 & 703.9 & Average residual Std. \\
\hline 75 & 2.25 & 262723 & 4748.4 & deviation of impurity-A = \\
\hline 100 & 3.0 & 355342 & 1011.8 & $\begin{array}{c}2275 \\
\end{array}$ \\
\hline 125 & 3.75 & 447581 & 2314.3 & $\begin{aligned} & \text { Slope }=3578 \\
\mathrm{LOD}= & 1.9 \%(0.057 \mu \mathrm{g} / \mathrm{mL})\end{aligned}$ \\
\hline 150 & 4.5 & 534962 & 2198.3 & $\mathrm{LOO}=6.4 \%(1.92 \mu \mathrm{g} / \mathrm{mL}$ \\
\hline 200 & 6.0 & 710570 & 5438.8 & \\
\hline
\end{tabular}


TABLE-3

ROBUSTNESS STUDY OF DAPIPRAZOLE AND IMPURITY-A

\begin{tabular}{lccccccc}
\hline \multicolumn{1}{c}{ Parameters studied } & Retention time & Peak area & Std. dev & RSD $(\%)$ & Difference (\%) & Limit (\%) & Resolution \\
\hline Flow decrease $(0.9 \mathrm{~mL} / \mathrm{min})$ & 12.896 & 52559 & 706.5 & 1.3 & 7.1 & 33 & 5.89 \\
Flow increase $(1.1 \mathrm{~mL} / \mathrm{min})$ & 10.802 & 49136 & 349.7 & 0.7 & 7.1 & 33 & 5.41 \\
Different column & 12.351 & 69282 & 888.6 & 1.3 & 21.4 & 33 & 7.18 \\
Different instrument & 11.819 & 66741 & 857 & 1.285 & 21.4 & 33 & 5.688 \\
\hline
\end{tabular}

component. The typical chromatogram of acid degradation study after $48 \mathrm{~h}$ indicates the presence of additional peak at 7.180 and 8.932 min with percent peak areas each of $0.02 \%$. This indicates the mild instability of dapiprazole in acid degradation studies. Otherwise, the drug was stable towards acidic hydrolysis and underwent no significant degradation. The chromatogram of $0 \mathrm{~h}$ sample in alkali stress induced study shows no degradation of dapiprazole indicates stability of the component. The typical chromatogram of dapiprazole in alkaline degradation studies after $48 \mathrm{~h}$ shows that the compound is moderately sensitive in base-stress studies. Four additional peaks have appeared in chromatogram at 7.1, 8.9, $10.4 \mathrm{~min}$ and at 11.578 consecutively apart from major peaks, indicates the mild base-induced degradation has occurred for dapiprazole in stress study. The two of additional peaks are eluting closely with process related impurity of dapiprazole. However, in oxidative stress study, the chromatogram of zero-hour sample shows neither degraded dapiprazole nor shifting in retention time, which indicates stability of the component in oxidation stress studies. The typical chromatogram of dapiprazole in peroxide induced degradation studies shows that the compound is extremely sensitive in oxidation studies. Degradation with hydrogen peroxide in oxidation stress resulted in eighteen additional peaks. This indicates the significant degradation of dapiprazole in peroxide induced stress study. In addition, predominant degradants were found with retention times of 22.4 and $4.1 \mathrm{~min}$ in typical chromatogram of oxidation stress study. However, the total peak area of all additional peaks collectively present in oxidative chromatogram is ca. $8 \%$ which indicates the extremely sensitive nature of dapiprazole in peroxide forced degradation study. Only $0.14 \%$ of dapiprazole $\mathrm{HCl}$ degraded indicating that the active ingredient is stable towards heat. Dapiprazole bulk sample appears to be stable in light induced stress study because the percent peak area of major peak is 99.91 . The collective peak areas of the additional peaks are $0.05 \%$, which indicates the stability of the dapiprazole in photodegradation studies.

\section{Conclusion}

The liquid chromatographic method with isocratic elution developed for the simultaneous determination of dapiprazole and its process related impurity A in the bulk drug, was fully validated and proved to be reliable, sensitive, accurate, precise and robust. The method has higher sensitivity towards the determination of impurities and it is the first time that such method appears in the literature and can be useful for routine analysis and quality control of dapiprazole in the relevant forms. The developed method was found to be accurate, precise, specific and linear. Thus, the method can be used for quality assurance of dapiprazole in bulk drugs and it can extend to validate the pharmaceutical formulations.

\section{ACKNOWLEDGEMENTS}

The authors are grateful to M/s Bioleo Analytical Labs India Pvt. Ltd., Hyderabad for the supply of dapiprazole and its process related impurity A as a gift sample. The authors also thankful to Q.S. Labs, Hyderabad for providing the necessary facilities to carry out the research work.

\section{REFERENCES}

1. R.W. Allinson, D.S. Gerber, S. Bieber and B.L. Hodes, Ann. Ophthalmol, 22, 131 (1990).

2. R.W. Allinson, Rev Eyes: Alpha Blocker to Reverse Diagnostic Mydriasis, Ocular Surgery News, p. 19 (1994).

3. L. Bonomi and G. Marchini, The Potential Interest of Alpha-Adrenergic Blocking Agents in Ophthalmology, pp. 1-6, (1987).

4. M.G. Bucci, D. D'Andrea, A. Bettini and M. De Gregorio, Glaucoma, 9, 94 (1987). 